\title{
THE BOHR INEQUALITY FOR THE GENERALIZED CÉSARO AVERAGING OPERATORS
}

\author{
ILGIZ R KAYUMOV, DIANA M. KHAMMATOVA AND SAMINATHAN PONNUSAMY
}

\begin{abstract}
The main aim of this paper is to prove a generalization of the classical Bohr theorem and as an application, we obtain a counterpart of Bohr theorem for the generalized Cesáro operator.
\end{abstract}

\section{Introduction AND PRELiminaries}

This work is connected with one of classical results known as Bohr's theorem for the class $\mathcal{B}$ of analytic self mappings of the unit disk $\mathbb{D}:=\{z \in \mathbb{C}:|z|<1\}$. Harold Bohr's initial result of 1914 has sharpened by several prominent mathematicians. Since then it has been a source of investigations in numerous other function spaces. The Bohr theorem in its final form says the following.

Theorem A. [11, H. Bohr, 1914] If $f \in \mathcal{B}$ and $f(z)=\sum_{n=0}^{\infty} a_{n} z^{n}$, then $\sum_{n=0}^{\infty}\left|a_{n}\right| r^{n} \leq 1$ for $r \leq 1 / 3$ and the constant $1 / 3$ cannot be improved.

The constant $1 / 3$ in this theorem is called the Bohr radius. Few other proofs of this result were also given (see [29]). It is also true [24] that $\sum_{n=0}^{\infty}\left|a_{n}\right|(1 / 3)^{n}=1$ if and only if $f$ is a constant function. However, there are a lot of generalizations and extensions of this theorem (cf. [12 14, 29]). The interest on this topic was revived due to the discovery of extensions to domains in $\mathbb{C}^{n}$ and to more general abstract setting in various contexts, due mainly to works of Aizenberg, Boas, Khavinson, and others (cf. [2 44, 8, 10, 25]). In [2, 4], multidimensional analogues of Bohr's inequality in which the unit disk $\mathbb{D}$ is replaced by a domain in $\mathbb{C}^{n}$ were considered. One can also find some information about it, for example, in the survey by Abu-Muhanna et al. 6], [15, Chapter 8] and the monograph 21.

Another widely discussed problem is the investigation of the asymptotical behaviour of the Bohr sum. In this connection, a natural question is to ask for the best constant $C(r) \geq 1$ such that for $f \in \mathcal{B}$ we have

$$
\sum_{n=0}^{\infty}\left|a_{n}\right| r^{n} \leq C(r) .
$$

2000 Mathematics Subject Classification. Primary: 30A10, 30B10; 30C62, 30H05, 31A05, 41A58; Secondary: 30C75, 40A30.

Key words and phrases. Bohr inequality, Bohr radius, convolution, Gaussian Hypergeometric functions, generalized Césaro operators.

The article is to appear in Mediterranean Journal of Mathematics.

File: arXiv 'General'Bohr 'GenCesara.tex, printed: 6-4-2021, 1.41. 
Indeed, Bombieri [12] proved that

$$
\sum_{n=0}^{\infty}\left|a_{n}\right| r^{n} \leq \frac{3-\sqrt{8\left(1-r^{2}\right)}}{r} \text { for } 1 / 3 \leq r \leq 1 / \sqrt{2} .
$$

Later in [13], Bombieri and Bourgain proved that

$$
\sum_{n=0}^{\infty}\left|a_{n}\right| r^{n}<\frac{1}{\sqrt{1-r^{2}}} \text { for } r>1 / \sqrt{2}
$$

so that $C(r) \asymp\left(1-r^{2}\right)^{-1 / 2}$ as $r \rightarrow 1$. In the same paper they also obtained a lower bound. Namely, they proved that for $\varepsilon>0$ there exists a constant $c=c(\varepsilon)$ such that

$$
\sum_{n=0}^{\infty}\left|a_{n}\right| r^{n} \geq\left(1-r^{2}\right)^{-1 / 2}-\left(c \log \frac{1}{1-r}\right)^{3 / 2+\varepsilon} \quad \text { as } r \rightarrow 1 .
$$

Some recent results on the topic can be found in [7, 17, 19, 22, 23, 26, 27].

The article is organized as follows. First, we consider a natural generalization of Theorem A and make it applicable to many situations (see Theorem 1). Secondly, in Section 3 as an application, we investigate a convolution counterpart of Bohr radius and also the operator counterpart of the so-called one parameter family of averaging Cesáro operator $\mathcal{C}_{f}^{1}$, discussed for example in [1,28]. Finally, in Theorem [6, we discuss asymptotic Bohr radius for $\mathcal{C}_{f}^{1}$

\section{BOHR RADIUS IN GENERAL FORM}

Let $\left\{\varphi_{k}(r)\right\}_{k=0}^{\infty}$ be a sequence of nonnegative continuous functions in $[0,1)$ such that the series $\sum_{k=0}^{\infty} \varphi_{k}(r)$ converges locally uniformly with respect to $r \in[0,1)$.

Theorem 1. Let $f \in \mathcal{B}, f(z)=\sum_{k=0}^{\infty} a_{k} z^{k}$ and $p \in(0,2]$. If

$$
\varphi_{0}(r)>\frac{2}{p} \sum_{k=1}^{\infty} \varphi_{k}(r) \quad \text { for } r \in[0, R),
$$

where $R$ is the minimal positive root of the equation

$$
\varphi_{0}(x)=\frac{2}{p} \sum_{k=1}^{\infty} \varphi_{k}(x)
$$

then the following sharp inequality holds:

$$
B_{f}(\varphi, p, r):=\left|a_{0}\right|^{p} \varphi_{0}(r)+\sum_{k=1}^{\infty}\left|a_{k}\right| \varphi_{k}(r) \leq \varphi_{0}(r) \text { for all } r \leq R .
$$

In the case when $\varphi_{0}(x)<(2 / p) \sum_{k=1}^{\infty} \varphi_{k}(x)$ in some interval $(R, R+\varepsilon)$, the number $R$ cannot be improved. If the functions $\varphi_{k}(x)(k \geq 0)$ are smooth functions then the last condition is equivalent to the inequality

$$
\varphi_{0}^{\prime}(R)<\frac{2}{p} \sum_{k=1}^{\infty} \varphi_{k}^{\prime}(R)
$$


Proof. For $f \in \mathcal{B}$, an application of Schwarz-Pick lemma gives the inequality $\left|a_{k}\right| \leq 1-|a|^{2}$ for all $k \geq 1$ and thus, we get that

$$
\begin{aligned}
B_{f}(\varphi, p, r) & \leq\left|a_{0}\right|^{p} \varphi_{0}(r)+\left(1-\left|a_{0}\right|^{2}\right) \sum_{k=1}^{\infty} \varphi_{k}(r) \\
& =\varphi_{0}(r)+\left(1-\left|a_{0}\right|^{2}\right)\left[\sum_{k=1}^{\infty} \varphi_{k}(r)-\left(\frac{1-\left|a_{0}\right|^{p}}{1-\left|a_{0}\right|^{2}}\right) \varphi_{0}(r)\right] \\
& \leq \varphi_{0}(r)+\left(1-\left|a_{0}\right|^{2}\right)\left[\sum_{k=1}^{\infty} \varphi_{k}(r)-\frac{p}{2} \varphi_{0}(r)\right] \\
& \leq \varphi_{0}(r), \text { by Eqn. (1) }
\end{aligned}
$$

for all $r \leq R$, by the definition of $R$. This proves the desired inequality (2). In the third inequality above, we have used the following fact:

$$
A(x)=\frac{1-x^{p}}{1-x^{2}} \geq \frac{p}{2} \text { for all } x \in[0,1)
$$

and there is nothing to prove for $p=2$. This inequality is easy to verify. Indeed,

$$
A^{\prime}(x)=-\frac{x M(x)}{\left(1-x^{2}\right)^{2}}, \quad M(x)=(2-p) x^{p}+p x^{p-2}-2,
$$

and, since $M^{\prime}(x)=-p(2-p) x^{p-3}\left(1-x^{2}\right)<0$ for $x \in(0,1)$ and for each $p \in(0,2)$, it follows that $M(x)>M(1)=0$ and thus, $A(x)$ is decreasing on $[0,1)$. Hence $A(x) \geq$ $\lim _{x \rightarrow 1^{-}} A(x)=p / 2$, as desired.

Now let us prove that $R$ is an optimal number. We consider the function

$$
f(z)=\frac{z-a}{1-a z}
$$

with $a \in[0,1)$. For this function we have

$$
\begin{aligned}
\left|a_{0}\right|^{p} \varphi_{0}(r)+\sum_{k=1}^{\infty}\left|a_{k}\right| \varphi_{k}(r)= & a^{p} \varphi_{0}(r)+\left(1-a^{2}\right) \sum_{k=1}^{\infty} a^{k-1} \varphi_{k}(r) \\
= & \varphi_{0}(r)+(1-a)\left[2 \sum_{k=1}^{\infty} a^{k-1} \varphi_{k}(r)-p \varphi_{0}(r)\right] \\
& -(1-a)\left[(1-a) \sum_{k=1}^{\infty} a^{k-1} \varphi_{k}(r)+\left(\frac{1-a^{p}}{1-a}-p\right) \varphi_{0}(r)\right] \\
= & \varphi_{0}(r)+(1-a)\left[2 \sum_{k=1}^{\infty} a^{k-1} \varphi_{k}(r)-p \varphi_{0}(r)\right]+O\left((1-a)^{2}\right)
\end{aligned}
$$

as $a \rightarrow 1^{-}$. Now it is easy to see that the last number is $>\varphi_{0}(r)$ when $a$ is close to 1 . The proof of the theorem is complete. 
Remark. Clearly for $p>2$, we see that $1 \leq A(x)<p / 2$ for $x \in[0,1)$, and thus, in this case, Theorem 1 holds by replacing the factor $2 / p$ in Eqn. (11) by 1 and also at the other three places in the statement. The most important cases are at $p=1,2$.

Example 1. Suppose that $f \in \mathcal{B}, f(z)=\sum_{k=0}^{\infty} a_{k} z^{k}$ and $p \in(0,2]$. Then Theorem 1 gives the following:

(1) For $\varphi_{k}(r)=r^{k}(k \geq 0)$, we easily have (see [9, Proposition 1.4] and [26, Remark 1])

$$
\left|a_{0}\right|^{p}+\sum_{k=1}^{\infty}\left|a_{k}\right| r^{k} \leq 1 \text { for } r \leq R_{1}(p)=\frac{p}{2+p}
$$

and the constant $R_{1}(p)$ cannot be improved. The case $p=1$ is the classical Bohr inequality. The case $p=2$ is due to [24] and the inequality in this case does play a special role. We remark that for $p>2, R_{1}(p)$ should be taken as $1 / 2$.

(2) For $\varphi_{k}(r)=(k+1) r^{k}(k \geq 0)$, we easily have the sharp inequality

$$
\left|a_{0}\right|^{p}+\sum_{k=1}^{\infty}(k+1)\left|a_{k}\right| r^{k} \leq 1 \text { for } r \leq R_{2}(p)=1-\sqrt{\frac{2}{2+p}} .
$$

(3) For $\varphi_{0}(r)=1$ and $\varphi_{k}(r)=k^{\alpha} r^{k}(k \geq 1)$, the condition (11) reduces to $p \geq$ $2 \sum_{k=1}^{\infty} k^{\alpha} r^{k}$. In particular, as

$$
\sum_{k=1}^{\infty} k r^{k}=\frac{r}{(1-r)^{2}} \text { and } \sum_{k=1}^{\infty} k^{2} r^{k}=\frac{r(1+r)}{(1-r)^{3}},
$$

it can be easily seen that the following sharp inequalities (with $\alpha=1,2$ ) hold:

$$
\left|a_{0}\right|^{p}+\sum_{k=1}^{\infty} k\left|a_{k}\right| r^{k} \leq 1 \text { for } r \leq R_{3}(p)=\frac{p+1-\sqrt{2 p+1}}{p}
$$

and

$$
\left|a_{0}\right|^{p}+\sum_{k=1}^{\infty} k^{2}\left|a_{k}\right| r^{k} \leq 1 \text { for } r \leq R_{4}(p),
$$

where $R_{4}(p)$ is the minimal positive root of the equation $p(1-r)^{3}-2 r(1+r)=0$.

\section{Convolution counterpart of Bohr Radius}

For two analytic functions $f(z)=\sum_{k=0}^{\infty} a_{k} z^{k}$ and $g(z)=\sum_{k=0}^{\infty} b_{k} z^{k}$ in $\mathbb{D}$, we define the Hadamard product (or convolution) $f * g$ of $f$ and $g$ by the power series

$$
(f * g)(z)=\sum_{k=0}^{\infty} a_{k} b_{k} z^{k}, \quad z \in \mathbb{D} .
$$

Clearly, $f * g=g * f$.

As an application of Theorem 1 we consider first the convolution operator of the form

$$
(F * f)(z)=\sum_{k=0}^{\infty} \gamma_{k} a_{k} z^{k}
$$


where $F(z):={ }_{2} F_{1}(a, b ; c ; z)=F(a, b ; c ; z)$ denotes the Gaussian hypergeometric function defined by the power series expansion

$$
F(z)=\sum_{k=0}^{\infty} \gamma_{k} z^{k}, \quad \gamma_{k}=\frac{(a)_{k}(b)_{k}}{(c)_{k}(1)_{k}}
$$

Clearly, $F(a, b ; c ; z)$ is analytic in $\mathbb{D}$ and in particular, $F(a, 1 ; 1 ; z)=(1-z)^{-a}$. Here $a, b, c$ are complex numbers such that $c \neq-m, m=0,1,2, \ldots$, and $(a)_{k}$ is the shifted factorial defined by Appel's symbol

$$
(a)_{k}:=a(a+1) \cdots(a+k-1)=\frac{\Gamma(a+k)}{\Gamma(a)}, \quad k \in \mathbb{N},
$$

and $(a)_{0}=1$ for $a \neq 0$. In the exceptional case $c=-m, m=0,1,2, \ldots, F(a, b ; c ; z)$ is defined if $a=-j$ or $b=-j$, where $j=0,1,2, \ldots$ and $j \leq m$. It is clear that if $a=-m$, a negative integer, then $F(a, b ; c ; z)$ becomes a polynomial of degree $m$ in $z$.

Theorem 2. Let $f(z)=\sum_{k=0}^{\infty} a_{k} z^{k}$ belong to $\mathcal{B}$ and $p \in(0,2]$. Assume that $a, b, c>-1$ such that all $\gamma_{k}$ have the same sign for $k \geq 0$. Then

$$
\left|a_{0}\right|^{p}+\sum_{k=1}^{\infty}\left|\gamma_{k}\right|\left|a_{k}\right| r^{k} \leq 1 \text { for all } r \leq R
$$

where $R$ is the minimal positive root of the equation $|F(a, b ; c ; x)-1|=p / 2$, and the number $R$ cannot be improved.

Proof. We apply Theorem 1. Set $\varphi_{k}(r)=\gamma_{k} r^{k}$ and remark that $\gamma_{0}=1$. Let us also note that all $\gamma_{k}$ have the same sign. Therefore, we have

$$
|F(a, b ; c ; r)-1|=\sum_{k=1}^{\infty} \varphi_{k}(r) .
$$

Now the statement of Theorem 1 concludes the proof.

Sometimes the Bohr radius can be found explicitly. For instance, let us set $b=c=1$. In this case, we have $F(z)=(1-z)^{-a}$ and hence,

$$
|F(a, b ; c ; r)-1|=(1-r)^{-a}-1=\frac{p}{2}, \text { i.e., } R=1-\left(\frac{2}{2+p}\right)^{1 / a}
$$

which in the cases $a=1$ and $p=1$ coincide with the classical Bohr radius. Note that the case $a=2$ is dealt also in Example 1(2).

\section{4. $\alpha$-CESÁRO OPERATORS}

For any $\alpha \in \mathbb{C}$ with $\operatorname{Re} \alpha>-1$, we consider

$$
\frac{1}{(1-z)^{\alpha+1}}=\sum_{k=0}^{\infty} A_{k}^{\alpha} z^{k}, \quad A_{k}^{\alpha}=\frac{(\alpha+1)_{n}}{(1)_{n}}
$$

Next, by comparing the coefficient of $z^{n}$ on both sides of the identity

$$
\frac{1}{(1-z)^{\alpha+1}} \cdot \frac{1}{1-z}=\frac{1}{(1-z)^{\alpha+2}},
$$


it follows that

$$
A_{n}^{\alpha+1}=\sum_{k=0}^{n} A_{k}^{\alpha}, \text { i.e., } \frac{1}{A_{n}^{\alpha+1}} \sum_{k=0}^{n} A_{n-k}^{\alpha}=1 .
$$

With this principle, the Cesáro operator of order $\alpha$ or $\alpha$-Cesáro operator (see Stempak [28]) on the space of analytic functions $f$ in the unit disk $\mathbb{D}$ is therefore defined by

$$
\mathcal{C}^{\alpha} f(z)=\sum_{n=0}^{\infty}\left(\frac{1}{A_{n}^{\alpha+1}} \sum_{k=0}^{n} A_{n-k}^{\alpha} a_{k}\right) z^{n},
$$

where $f(z)=\sum_{k=0}^{\infty} a_{k} z^{k}$. In terms of convolution, we can write this as

$$
\mathcal{C}^{\alpha} f(z)=\frac{f(z)}{(1-z)^{\alpha+1}} * F(1,1 ; \alpha+2 ; z)
$$

and thus, we have (cf. [1,28]) the following integral form

$$
\mathcal{C}^{\alpha} f(z)=(\alpha+1) \int_{0}^{1} f(t z) \frac{(1-t)^{\alpha}}{(1-t z)^{\alpha+1}} d t
$$

where $\operatorname{Re} \alpha>-1$. This for $\alpha=0$ gives

$$
\mathcal{C}^{0} f(z)=\sum_{n=0}^{\infty}\left(\frac{1}{n+1} \sum_{k=0}^{n} a_{k}\right) z^{n}
$$

which is simply the classical Cesáro operator considered by Hardy-Littlewood in 1932 [16]. Several authors have studied the boundedness property of these operators on different function spaces (see, for example, [5]). In [20], the present authors established a theorem giving an analog of Bohr theorem for the classical Cesáro operator $\mathcal{C}^{0} f$. It also considered the asymptotical behaviour of the Bohr sum in this case.

For $f \in \mathcal{B}$, we define a counterpart of Bohr sum for $\alpha>-1$ as

$$
\mathcal{C}_{f}^{\alpha}(r):=\sum_{n=0}^{\infty}\left(\frac{1}{A_{n}^{\alpha+1}} \sum_{k=0}^{n} A_{n-k}^{\alpha}\left|a_{k}\right|\right) r^{n} .
$$

Before writing a counterpart of Bohr theorem we need the following estimate.

Theorem 3. For $f \in \mathcal{B}$ and $\alpha>-1$, we have

$$
\left|\mathcal{C}^{\alpha}(f)(z)\right| \leq(\alpha+1) \Phi(r, 1, \alpha+1)=\frac{\alpha+1}{r^{\alpha+1}} \int_{0}^{r} \frac{t^{\alpha}}{1-t} d t
$$

where $\Phi(z, s, a)=\sum_{n=0}^{\infty} z^{n}(n+a)^{-s}$ is the Lerch transcendent function.

Proof. We may represent $\mathcal{C}^{\alpha}(f)$ as

$$
\mathcal{C}^{\alpha}(f)(z)=(\alpha+1) \int_{0}^{1} \frac{1}{(t-1) z+1} f\left(\frac{t z}{(t-1) z+1}\right)(1-t)^{\alpha} d t
$$


and thus,

$$
\begin{aligned}
\left|\mathcal{C}^{\alpha}(f)(z)\right| & \leq(\alpha+1) \int_{0}^{1} \frac{(1-t)^{\alpha}}{|(t-1) z+1|}\left|f\left(\frac{t z}{(t-1) z+1}\right)\right| d t \\
& \leq(\alpha+1) \int_{0}^{1} \frac{(1-t)^{\alpha}}{(t-1) r+1} d t=\mathcal{C}_{f_{0}}^{\alpha}(r), \quad f_{0}(z)=1 .
\end{aligned}
$$

This integral is not easy to calculate and therefore, we may return to the standard series representation and obtain

$$
\mathcal{C}_{f_{0}}^{\alpha}(r)=\sum_{n=0}^{\infty} \frac{A_{n}^{\alpha}}{A_{n}^{\alpha+1}} r^{n}=(\alpha+1) \sum_{n=0}^{\infty} \frac{r^{n}}{\alpha+n+1},
$$

and the proof is complete.

Now we are ready to prove the counterpart of Bohr theorem for the $\alpha$-Cesáro operator.

Theorem 4. Let $f(z)=\sum_{k=0}^{\infty} a_{k} z^{k}$ belong to $\mathcal{B}$ and $\alpha>-1$. Then

$$
\mathcal{C}_{f}^{\alpha}(r) \leq(\alpha+1) \sum_{n=0}^{\infty} \frac{r^{n}}{n+\alpha+1}=\frac{\alpha+1}{r^{\alpha+1}} \int_{0}^{r} \frac{t^{\alpha}}{1-t} d t \text { for all } r \leq R,
$$

where $R=R(\alpha)$ is the minimal positive root of the equation

$$
3(1+\alpha) \sum_{n=0}^{\infty} \frac{x^{n}}{n+\alpha+1}=\frac{2}{1-x}, \quad \text { i.e., } \quad \sum_{n=0}^{\infty} \frac{\alpha+1-2 n}{n+\alpha+1} x^{n}=0 .
$$

The number $R$ cannot be replaced by a larger constant. Note that $R(0)=0.5335 \ldots$.

Proof. We apply Theorem 1 with $p=1$. If we write

$$
\mathcal{C}^{\alpha}(f)(z)=\sum_{n=0}^{\infty} a_{n} \phi_{n}(z)
$$

then collecting the terms involving only $a_{n}$ in the right hand side of (4) we find that

$$
\phi_{n}(z)=\sum_{k=n}^{\infty} \frac{A_{k-n}^{\alpha}}{A_{k}^{\alpha+1}} z^{k}
$$

so that for the $\alpha$-Cesáro operators $\mathcal{C}^{\alpha}(f)$ we have

$$
\varphi_{0}(x)=\sum_{k=0}^{\infty} \frac{A_{k}^{\alpha}}{A_{k}^{\alpha+1}} x^{k}=(\alpha+1) \sum_{k=0}^{\infty} \frac{x^{k}}{k+\alpha+1}, \quad x \in[0,1),
$$

by the definition of $A_{k}^{\alpha}$. Moreover, by setting $f(z)=1 /(1-z)$ in (6) $)$, it is not difficult to find from (3) and (4) that

$$
\sum_{n=0}^{\infty} \varphi_{n}(r)=C^{\alpha}\left(\frac{1}{1-r}\right)=\frac{1}{1-r}
$$


and thus, Eqn. (11) for $p=1$ takes the form

$$
3 \phi_{0}(r)>2 \sum_{n=0}^{\infty} \varphi_{n}(r) \text {, i.e., } 3(1+\alpha) \sum_{n=0}^{\infty} \frac{r^{n}}{n+\alpha+1}>\frac{2}{1-r} .
$$

The desired inequality (5) follows from Theorem 1 and the sharpness part also follows.

In what follows ${ }_{p} F_{q}$ represent the generalized hypergeometric function defined by

$$
{ }_{p} F_{q}\left(a_{1}, \ldots, a_{p} ; c_{1}, \ldots, c_{q} ; z\right)=\sum_{n=0}^{\infty} \frac{\left(a_{1}\right)_{n} \cdots\left(a_{p}\right)_{n}}{\left(c_{1}\right)_{n} \cdots\left(c_{q}\right)_{n}} \frac{z^{n}}{n !} .
$$

We remark that in the interesting case where $p=q+1$, the series converges for $|z|<1$. If $\operatorname{Re}\left(\sum_{j=1}^{q} c_{j}-\sum_{j=1}^{q+1} a_{j}\right)>0$, then ${ }_{q+1} F_{q}$ converges also at the point $z=1$.

Theorem 5. For $f \in \mathcal{B}$ and $\alpha>-1$, the inequality $\mathcal{C}_{f}^{\alpha}(r) \leq S_{\alpha}(r)$ holds for all $r \in[0,1)$, where $S_{\alpha}(r)$ is equal to

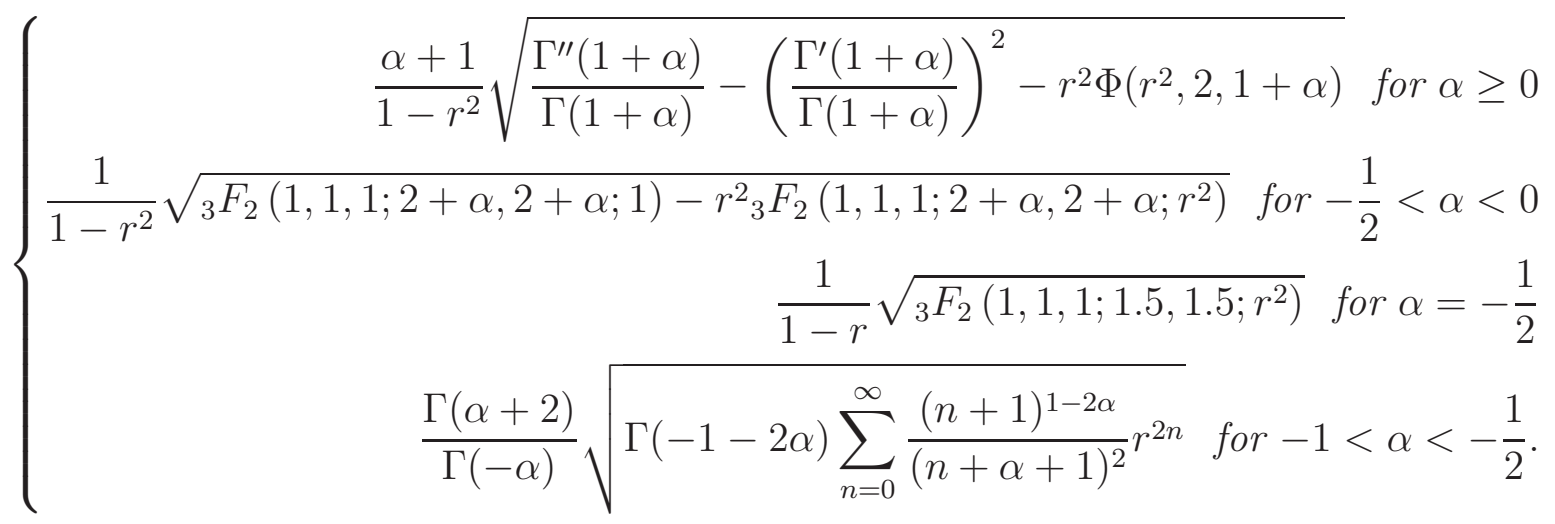

Here $\Phi(z, s, a)$ is the Lerch transcendent function.

Proof. First of all, we represent $\mathcal{C}_{f}^{\alpha}(r)$ as

$$
\mathcal{C}_{f}^{\alpha}(r)=\sum_{n=0}^{\infty} \frac{1}{A_{n}^{\alpha+1}}\left(\sum_{k=0}^{n} A_{n-k}^{\alpha}\left|a_{k}\right|\right) r^{n}=\sum_{n=0}^{\infty}\left|a_{n}\right| \phi_{n}(r),
$$

where $\phi_{n}(r)$ is defined by (7). Using the triangle inequality and the fact that $\sum_{n=0}^{\infty}\left|a_{n}\right|^{2} \leq$ 1 (for $f \in \mathcal{B}$ ), we can estimate

$$
\mathcal{C}_{f}^{\alpha}(r) \leq \sqrt{\sum_{n=0}^{\infty}\left|a_{n}\right|^{2}} \cdot \sqrt{\sum_{n=0}^{\infty} \phi_{n}^{2}(r)} \leq \sqrt{\sum_{n=0}^{\infty} \phi_{n}^{2}(r)} .
$$

For $\alpha>-1 / 2$, we use the triangle inequality one more time and obtain

$$
\phi_{n}^{2}(r)=\left(\sum_{k=n}^{\infty} \frac{A_{k-n}^{\alpha}}{A_{k}^{\alpha+1}} r^{k}\right)^{2} \leq \sum_{k=n}^{\infty}\left(\frac{A_{k-n}^{\alpha}}{A_{k}^{\alpha+1}}\right)^{2} \cdot \sum_{k=n}^{\infty} r^{2 k}=\frac{r^{2 n}}{1-r^{2}} \sum_{k=n}^{\infty}\left(\frac{A_{k-n}^{\alpha}}{A_{k}^{\alpha+1}}\right)^{2} .
$$

To estimate the second term on the right, we first observe that

$$
A_{k}^{\alpha}=\left(\frac{k+1}{\alpha+k+1}\right) A_{k+1}^{\alpha} \text { for all } \alpha>-1 \text { and } k \geq 0 .
$$


First we see that $A_{k}^{\alpha} \leq A_{k+1}^{\alpha}$ for all $\alpha \geq 0$ and $k \geq 0$. As a consequence, one can see that for $\alpha>0$ and for $k \geq n$,

$$
\frac{A_{k-n}^{\alpha}}{A_{k}^{\alpha+1}} \leq \frac{A_{k}^{\alpha}}{A_{k}^{\alpha+1}}=\frac{1+\alpha}{1+k+\alpha}
$$

which gives

$$
\phi_{n}^{2}(r) \leq \frac{r^{2 n}}{1-r^{2}} \sum_{k=n}^{\infty} \frac{(\alpha+1)^{2}}{(1+k+\alpha)^{2}}
$$

so that

$$
\begin{aligned}
\sum_{n=0}^{\infty} \phi_{n}^{2}(r) & \leq \frac{(\alpha+1)^{2}}{1-r^{2}} \sum_{n=0}^{\infty} r^{2 n} \sum_{k=n}^{\infty} \frac{1}{(1+k+\alpha)^{2}} \\
& =\frac{(\alpha+1)^{2}}{1-r^{2}} \sum_{k=0}^{\infty} \frac{1}{(1+k+\alpha)^{2}} \sum_{n=0}^{k} r^{2 n} \\
& =\frac{(\alpha+1)^{2}}{\left(1-r^{2}\right)^{2}} \sum_{k=0}^{\infty} \frac{1-r^{2(k+1)}}{(1+k+\alpha)^{2}} \\
& =\frac{(\alpha+1)^{2}}{\left(1-r^{2}\right)^{2}}\left(\frac{\Gamma^{\prime \prime}(1+\alpha)}{\Gamma(1+\alpha)}-\left(\frac{\Gamma^{\prime}(1+\alpha)}{\Gamma(1+\alpha)}\right)^{2}-r^{2} \Phi\left(r^{2}, 2,1+\alpha\right)\right)
\end{aligned}
$$

Secondly, by (8), we find that $A_{k}^{\alpha} \geq A_{k+1}^{\alpha}$ for all $-1<\alpha<0$ and $k \geq 0$, and thus,

$$
\frac{A_{k-n}^{\alpha}}{A_{k}^{\alpha+1}} \leq \frac{A_{0}^{\alpha}}{A_{k}^{\alpha+1}}=\frac{1}{A_{k}^{\alpha+1}}=\frac{(1)_{k}}{(\alpha+2)_{k}}
$$

which gives

$$
\phi_{n}^{2}(r) \leq \frac{r^{2 n}}{1-r^{2}} \sum_{k=n}^{\infty}\left(\frac{(1)_{k}}{(\alpha+2)_{k}}\right)^{2} .
$$

Hence, as before, we can easily deduce that

$$
\begin{aligned}
\sum_{n=0}^{\infty} \phi_{n}^{2}(r) & \leq \frac{1}{\left(1-r^{2}\right)^{2}} \sum_{k=0}^{\infty}\left(\frac{(1)_{k}}{(\alpha+2)_{k}}\right)^{2}\left(1-r^{2(k+1)}\right) \\
& =\frac{1}{\left(1-r^{2}\right)^{2}}\left({ }_{3} F_{2}(1,1,1 ; 2+\alpha, 2+\alpha ; 1)-r^{2}{ }_{3} F_{2}\left(1,1,1 ; 2+\alpha, 2+\alpha ; r^{2}\right)\right)
\end{aligned}
$$

and the last expression converges for $-0.5<\alpha<0$.

Thirdly, for $\alpha=-0.5$ we use the inequality (10) and obtain

$$
\frac{A_{k-n}^{-1 / 2}}{A_{k}^{1 / 2}} \leq \frac{A_{0}^{-1 / 2}}{A_{n}^{1 / 2}}=\frac{1}{A_{n}^{1 / 2}}=\frac{(1)_{n}}{(3 / 2)_{n}}=\frac{\sqrt{\pi} \Gamma(n+1)}{2 \Gamma(n+3 / 2)} .
$$


In this case

$$
\begin{aligned}
\sum_{n=0}^{\infty} \phi_{n}^{2}(r) & \leq \sum_{n=0}^{\infty} \frac{\pi \Gamma^{2}(n+1)}{4 \Gamma^{2}(n+1.5)}\left(\sum_{k=n}^{\infty} r^{k}\right)^{2}=\frac{\pi}{4(1-r)^{2}} \sum_{n=0}^{\infty} \frac{\Gamma^{2}(n+1)}{\Gamma^{2}(n+1.5)} r^{2 n} \\
& =\frac{1}{(1-r)^{2}}{ }_{3} F_{2}\left(1,1,1 ; 1.5,1.5 ; r^{2}\right)
\end{aligned}
$$

Finally, for $\alpha<-0.5$, we write the quotient as

$$
\frac{A_{k-n}^{\alpha}}{A_{k}^{\alpha+1}}=\frac{(\alpha+1)_{k-n}}{(1)_{k-n}} \cdot \frac{(1)_{k}}{(\alpha+2)_{k}}
$$

and estimate $\phi_{n}^{2}(r)$ using the triangle inequality in the following way:

$$
\begin{aligned}
\phi_{n}^{2}(r) & =\left(\sum_{k=n}^{\infty} \frac{(\alpha+1)_{k-n}}{(1)_{k-n}} \cdot \frac{(1)_{k}}{(\alpha+2)_{k}} r^{k}\right)^{2} \\
& \leq \sum_{k=n}^{\infty}\left(\frac{(\alpha+1)_{k-n}}{(1)_{k-n}}\right)^{2} \sum_{k=n}^{\infty}\left(\frac{(1)_{k}}{(\alpha+2)_{k}}\right)^{2} r^{2 k} \\
& ={ }_{2} F_{1}(\alpha+1, \alpha+1 ; 1 ; 1) \sum_{k=n}^{\infty}\left(\frac{(1)_{k}}{(\alpha+2)_{k}}\right)^{2} r^{2 k}
\end{aligned}
$$

where the first sum in the second step converges to

$$
{ }_{2} F_{1}(\alpha+1, \alpha+1 ; 1 ; 1)=\frac{\Gamma(-1-2 \alpha)}{\Gamma^{2}(-\alpha)} \text { for }-1<\alpha<-0.5 .
$$

Here we have used the well-known formula

$$
{ }_{2} F_{1}(a, b ; c ; 1)=\frac{\Gamma(c) \Gamma(c-a-b)}{\Gamma(c-a) \Gamma(c-b)}<\infty \text { for } c>a+b .
$$

To estimate the other series, we use the well-known inequality

$$
\frac{(1)_{n}}{(s)_{n}} \leq \frac{\Gamma(s)}{(n+1)^{s-1}},
$$

which holds for any natural $n$ and $0 \leq s \leq 1$. Therefore,

$$
\frac{(1)_{k}}{(\alpha+2)_{k}}=\left(\frac{\alpha+1}{\alpha+k+1)}\right) \frac{(1)_{k}}{(\alpha+1)_{k}} \leq \frac{\Gamma(\alpha+2)}{\alpha+k+1)} \frac{1}{(k+1)^{\alpha}} .
$$

It follows that

$$
\begin{aligned}
\sum_{n=0}^{\infty} \phi_{n}^{2}(r) & \leq{ }_{2} F_{1}(\alpha+1, \alpha+1 ; 1 ; 1) \Gamma^{2}(\alpha+2) \sum_{n=0}^{\infty} \sum_{k=n}^{\infty} \frac{(k+1)^{-2 \alpha}}{(k+\alpha+1)^{2}} r^{2 k} \\
& \leq \frac{\Gamma(-1-2 \alpha) \Gamma^{2}(\alpha+2)}{\Gamma^{2}(-\alpha)} \sum_{n=0}^{\infty} \frac{(n+1)^{1-2 \alpha}}{(n+\alpha+1)^{2}} r^{2 n} .
\end{aligned}
$$

This finishes the proof. 


\section{Asymptotic Bohr Radius For $\mathcal{C}_{f}^{1}$}

Let us study the order of the estimate for $\alpha=1$. We recall the following equality:

$$
\operatorname{Li}_{2}(x)+\operatorname{Li}_{2}(1-x)=\frac{\pi^{2}}{6}-\log x \log (1-x) .
$$

Then the estimate looks like

$$
\frac{1}{1-r^{2}} \cdot 2 \sqrt{\frac{\pi^{2}}{6}-\frac{\operatorname{Li}_{2}\left(r^{2}\right)}{r^{2}}}=\frac{2 \sqrt{\frac{\pi^{2}}{6}-\frac{1}{r^{2}}\left(\frac{\pi^{2}}{6}-2 \log r \log \left(1-r^{2}\right)-\mathrm{Li}_{2}\left(1-r^{2}\right)\right)}}{1-r^{2}},
$$

where $\operatorname{Li}_{2}(z)=\sum_{k=1}^{\infty}\left(1 / k^{2}\right) z^{k}$ is a polylogarithm function. Moreover,

$$
\operatorname{Li}_{2}\left(1-r^{2}\right) \rightarrow 0 \text { and } \frac{\log (1 / r)}{1-r} \rightarrow 1 \text { as } r \rightarrow 1,
$$

and so we obtain

$$
\mathcal{C}_{f}^{1}(r) \leq \frac{2 \sqrt{2 \log r \log \left(1-r^{2}\right)}+o(1)}{1-r^{2}} \sim \sqrt{2} \cdot \sqrt{\frac{\log \frac{1}{1-r^{2}}}{1-r}} .
$$

We shall prove

Theorem 6. There exists an $f \in \mathcal{B}$ such that

$$
\mathcal{C}_{f}^{1}(r) \sim \frac{4 \sqrt{2 q}}{(3+q) \sqrt{1-r}} \approx \frac{1.47217 \ldots}{\sqrt{1-r}},
$$

where $q \approx 7.57736 \ldots$ is the root of the equation $3 q=(3+q) \log (1+q)$.

Proof. Consider the function $\phi_{n}(r)$ defined by (7) with $\alpha=1$ and calculate it using definition of $A_{k}^{\alpha}$. This gives that

$$
\phi_{n}(r)=\sum_{k=n}^{\infty} \frac{A_{k-n}^{1}}{A_{k}^{2}} r^{k}=2 \sum_{k=n}^{\infty} \frac{k-n+1}{(k+2)(k+1)} r^{k} .
$$

As

$$
\frac{r^{k+2}}{(k+2)(k+1)}=\int_{0}^{r} \int_{0}^{\rho} s^{k} d s d \rho
$$

it follows that

$$
\phi_{n}(r)=\frac{2}{r^{2}} \int_{0}^{r} \int_{0}^{\rho} s^{n} \sum_{k=n}^{\infty}(k-n+1) s^{k-n} d s d \rho=\frac{2}{r^{2}} \int_{0}^{r} \int_{0}^{\rho} \frac{s^{n}}{(1-s)^{2}} d s d \rho .
$$

In [13], Bombieri and Bourgain showed how one can build functions $h(z)=\sum_{k=0}^{\infty} h_{k} z^{k}$ and $f(z)=\sum_{k=0}^{\infty} a_{k} z^{k}$ in the family $\mathcal{B}$ such that
(i) $\left|h_{k}\right|=t^{k} \sqrt{1-t^{2}}$
(ii) $\|f-h\|_{2}:=\sqrt{\sum_{k=0}^{\infty}\left|h_{k}-a_{k}\right|^{2}}<\sqrt{1-t^{2}} \sqrt{\log \frac{1}{1-t}}$, 
where $0 \leq t \leq 1$ is some number.

Accordingly, using their idea, we obtain the following estimate

$$
\begin{aligned}
\mathcal{C}_{f}^{1}(r) & =\sum_{k=0}^{\infty}\left|h_{k}+a_{k}-h_{k}\right| \phi_{k}(r) \geq \sum_{k=0}^{\infty}\left|h_{k}\right| \phi_{k}(r)-\sum_{k=0}^{\infty}\left|h_{k}-a_{k}\right| \phi_{k}(r) \\
& \geq \sqrt{1-t^{2}} \sum_{k=0}^{\infty} t^{k} \phi_{k}(r)-\sqrt{1-t^{2}} \sqrt{\log \frac{1}{1-t}} \sqrt{\sum_{k=0}^{\infty} \phi_{k}^{2}(r)} .
\end{aligned}
$$

Using (11) and the above consideration, the first term in (12) can be calculated directly. Note that

$$
\sum_{k=0}^{\infty} t^{k} \phi_{k}(r)=\frac{2}{r^{2}} \sum_{k=0}^{\infty} \int_{0}^{r} \int_{0}^{\rho} \frac{t^{k} s^{k}}{(1-s)^{2}} d s d \rho=\frac{2}{r^{2}} \int_{0}^{r} \int_{0}^{\rho} \frac{d s d \rho}{(1-s)^{2}(1-t s)}
$$

and so to computet the integral on the right, we write

$$
\frac{1}{(1-s)^{2}(1-t s)}=\frac{1}{1-t} \cdot \frac{1}{(1-s)^{2}}-\frac{t}{(1-t)^{2}} \cdot \frac{1}{1-s}+\frac{t^{2}}{(1-t)^{2}} \cdot \frac{1}{1-t s},
$$

which gives by integration

$$
\int_{0}^{\rho} \frac{d s}{(1-s)^{2}(1-t s)}=\frac{1}{1-t} \cdot \frac{\rho}{1-\rho}+\frac{t}{(1-t)^{2}}[\log (1-\rho)-\log (1-t \rho)]
$$

and hence we can easily obtain by integrating it again

$$
\int_{0}^{r} \int_{0}^{\rho} \frac{d s d \rho}{(1-s)^{2}(1-t s)}=\frac{2[-r(1-t)+(1-r t)(\log (1-r t)-\log (1-r))]}{r^{2}(1-t)^{2}} .
$$

The second term in (12) can be estimated using the result from the first part of Theorem 51, i.e., Eqn. (9). For $\alpha=1$, we have

$$
\sqrt{\sum_{k=0}^{\infty} \phi_{k}^{2}(r)} \leq \frac{2}{1-r^{2}} \sqrt{\frac{\Gamma^{\prime \prime}(2)}{\Gamma(2)}-\left(\frac{\Gamma^{\prime}(2)}{\Gamma(2)}\right)^{2}-r^{2} \Phi\left(r^{2}, 2,2\right)}=\frac{2}{1-r^{2}} \sqrt{\frac{\pi^{2}}{6}-\frac{\mathrm{Li}_{2}\left(r^{2}\right)}{r^{2}}} .
$$

Therefore, the above discussion shows that $\mathcal{C}_{f}^{1}(r)$ is not less than

$$
\begin{aligned}
& \frac{1}{\sqrt{1-r}}\left(\frac{2 \sqrt{1-t^{2}} \sqrt{1-r}[-r(1-t)+(1-r t)(\log (1-r t)-\log (1-r))]}{r^{2}(1-t)^{2}}-\right. \\
& \left.-2 \sqrt{1-t^{2}} \sqrt{\log \frac{1}{1-t}} \cdot \frac{1}{\sqrt{1-r}(1+r)} \sqrt{\frac{\pi^{2}}{6}-\frac{\operatorname{Li}_{2}\left(r^{2}\right)}{r^{2}}}\right) .
\end{aligned}
$$

Let $t=r^{q}$ for some $q$. Since

$$
\lim _{r \rightarrow 1} \sqrt{1-r^{2 q}} \sqrt{\log \frac{1}{1-r^{q}}} \cdot \frac{1}{\sqrt{1-r}} \sqrt{\frac{\pi^{2}}{6}-\frac{\operatorname{Li}_{2}\left(r^{2}\right)}{r^{2}}}=0,
$$


we have to consider only the first term

$$
\frac{1}{\sqrt{1-r}} \cdot \frac{2 \sqrt{1+r}[-r+(1+r) \log (1+r)]}{r^{2}} \sim \frac{2 \sqrt{2}(\log 4-1)}{\sqrt{1-r}} \approx \frac{1.09261 \ldots}{\sqrt{1-r}} .
$$

Next, we calculate the limit

$$
\begin{aligned}
\lim _{r \rightarrow 1} \frac{2 \sqrt{1-r^{2 q}} \sqrt{1-r}\left[-r\left(1-r^{q}\right)+\left(1-r^{q+1}\right)\left(\log \left(1-r^{q+1}\right)\right.\right.}{r^{2}\left(1-r^{q}\right)^{2}} & \log (1-r))] \\
= & =\frac{-q+(q+1) \log (q+1)}{(q / 2)^{\frac{3}{2}}} .
\end{aligned}
$$

The point of maximum is the root of the equation $3 q=(3+q) \log (1+q)$. Calculation shows that $q \approx 7.57736 \ldots$ Therefore,

$$
\mathcal{C}_{f}^{1}(r) \sim \frac{4 \sqrt{2 q}}{(3+q) \sqrt{1-r}} \approx \frac{1.47217 \ldots}{\sqrt{1-r}}
$$

Acknowledgments. The work of I. Kayumov and D. Khammatova is supported by the Russian Science Foundation under grant 18-11-00115.

\section{REFERENCES}

1. M. R. Agrawal, P. Howlett, St. Lucas, S. Naik and S. Ponnusamy, Boundedness of generalized Césaro averaging operators on certain function spaces, J. Comput. Appl. Math. 180 (2005), 333-344.

2. L. Aizenberg, Multidimensional analogues of Bohr's theorem on power series, Proc. Amer. Math. Soc., 128(4) (2000), 1147-1155.

3. L. Aizenberg, Generalization of Caratheodory's inequality and the Bohr radius for multidimensional power series, Oper. Theory: Adv. Appl., 158 (2005), 87-94.

4. L. Aizenberg, A. Aytuna and P. Djakov, Generalization of a theorem of Bohr for basis in spaces of holomorphic functions of several complex variables, J. Math. Anal. Appl. 258(2) (2001), 429-447.

5. A. A. Albanese, J. Bonet, J.W. Ricker, The Cesàro operator on power series spaces, Studia Mathematica, 240 (2018), 47-68.

6. R. M. Ali, Y. Abu-Muhanna, and S. Ponnusamy, On the Bohr inequality, Progress in Approximation Theory and Applicable Complex Analysis (Edited by N.K. Govil et al. ), Springer Optimization and Its Applications, 117 (2016), 265-295.

7. R. M. Ali, R. W. Barnard and A. Yu. Solynin, A note on the Bohr's phenomenon for power series, J. Math. Anal. Appl. 449(1) (2017), 154-167.

8. C. Bénéteau, A. Dahlner and D. Khavinson, Remarks on the Bohr phenomenon, Comput. Methods Funct. Theory 4(1) (2004), 1-19.

9. O. Blasco, The Bohr radius of a Banach space, In Vector measures, integration and related topics, 5964, Oper. Theory Adv. Appl., 201, Birkhäuser Verlag, Basel, 2010.

10. H. P. Boas and D. Khavinson, Bohr's power series theorem in several variables, Proc. Amer. Math. Soc. 125(10) (1997), 2975-2979.

11. H. Bohr, A theorem concerning power series, Proc. London Math. Soc. 13(2) (1914), 1-5.

12. E. Bombieri, Sopra un teorema di H. Bohr e G. Ricci sulle funzioni maggioranti delle serie di potenze, Boll. Unione Mat. Ital. 17 (1962), 276-282.

13. E. Bombieri and J. Bourgain, A remark on Bohr's inequality, IMRN International Mathematics Research Notices, 80 (2004), 4307-4330. 
14. P. B. Djakov and M. S. Ramanujan, A remark on Bohr's theorems and its generalizations, J. Analysis 8 (2000), 65-77.

15. S. R. Garcia, J. Mashreghi and W. T. Ross, Finite Blaschke products and their connections, Springer, Cham, 2018.

16. G. H. Hardy and J. E. Littlewood, Some properties of fractional integrals II, Math. Z., 34(1932), 403-439.

17. A. Ismagilov, I. Kayumov, S. Ponnusamy, Sharp Bohr type inequality, J. Math. Anal. Appl. 489(1) (2020), 10 pages; Article124147

18. I. R. Kayumov and S. Ponnusamy, Bohr inequality for odd analytic functions, Comput. Methods Funct. Theory 17 (2017), 679-688.

19. I. R. Kayumov and S. Ponnusamy, Improved version of Bohr's inequality, C. R. Math. Acad. Sci. Paris 356(3) (2018), 272-277.

20. I R. Kayumov, D. M. Khammatova, and S. Ponnusamy, On the Bohr inequality for the Cesaro operator, C. R. Math. Acad. Sci. Paris 358(5)(2020), 615-620.

21. G. Kresin, and V. Maz'ya, Sharp real-part theorems. A unified approach, Translated from the Russian and edited by T. Shaposhnikova. Lecture Notes in Mathematics, 1903. Springer, Berlin, 200\%. xvi+140 pp.

22. G. Liu, Z. H. Liu and S. Ponnusamy, Refined Bohr inequality for bunded analytic functions, 16 pages; Preprint in: arXiv:2006.08930

23. M. S. Liu, Y. M. Shang and J. F. Xu, Bohr-type inequalities of analytic functions, J. Inequal. Appl., 345 (2018), 13 pp.

24. V. I. Paulsen, G. Popescu and D. Singh, On Bohr's inequality, Proc. London Math. Soc. 85(2) (2002), 493-512.

25. V. I. Paulsen and D. Singh, Bohrś inequality for uniform algebras, Proc. Amer. Math. Soc. 132(12)(2004), 3577-3579,

26. S. Ponnusamy, R. Vijayakumar and K.-J. Wirths, Improved Bohr's phenomenon in quasisubordination classes, Preprint. https://arxiv.org/pdf/1909.00780v2.pdf

27. S. Ponnusamy, R. Vijayakumar and K.-J. Wirths, New inequalities for the coefficients of unimodular bounded functions, Results Math. 75, 107 (2020). https://doi.org/10.1007/s00025-020-01240-1

28. K. Stempak, Cesáro averaging operators, Proc. Royal Soc. of Edinburgh, 124A(1994), 121-126.

29. M. Tomić, Sur un théorème de H. Bohr, Math. Scand. 11 (1962), 103-106.

I. R Kayumov, Kazan Federal University, 420008 Kazan, Russia

Email address: ikayumov@gmail.com

D. M. Khammatova, Kazan Federal University, 420008 Kazan, Russia

Email address: dianalynx@rambler.ru

S. Ponnusamy, Department of Mathematics, Indian Institute of Technology Madras, Chennai-600 036, India

Email address: samy@iitm.ac.in 